\title{
BIVARIATE ORDER POLYNOMIALS
}

\author{
MATTHIAS BECK, MARYAM FARAHMAND, GINA KARUNARATNE, AND SANDRA ZUNIGA RUIZ
}

\begin{abstract}
Motivated by Dohmen-Pönitz-Tittmann's bivariate chromatic polynomial $\chi_{G}(x, y)$, which counts all $x$-colorings of a graph $G$ such that adjacent vertices get different colors if they are $\leq y$, we introduce a bivarate version of Stanley's order polynomial, which counts order preserving maps from a given poset to a chain. Our results include decomposition formulas in terms of linear extensions, a combinatorial reciprocity theorem, and connections to bivariate chromatic polynomials.
\end{abstract}

\section{INTRODUCTION}

Graph coloring problems are ubiquitous in many areas within and outside of mathematics. The motivation of our study is the bivariate chromatic polynomial $\chi_{G}(x, y)$ of a graph $G=(V, E)$, first introduced in [4] and defined as the counting function of colorings $c: V \rightarrow[x]:=\{1,2, \ldots, x\}$ that satisfy for any edge $v w \in E$

$$
c(v) \neq c(w) \quad \text { or } \quad c(v)=c(w)>y .
$$

The usual univariate chromatic polynomial of $G$ can be recovered as the special evaluation $\chi_{G}(x, x)$. Dohmen, Pönitz, and Tittmann provided basic properties of $\chi_{G}(x, y)$ in [4], including polynomiality and special evaluations yielding the matching and independence polynomials of $G$. Subsequent results include a deletioncontraction formula and applications to Fibonacci-sequence identities [5], common generalizations of $\chi_{G}(x, y)$ and the Tutte polynomial [1], and closed formulas for paths and cycles [3].

For a finite poset $(P, \preceq)$, Stanley [6] (see also [8, Chapter 3]) famously introduced the "chromatic-like" order polynomial $\Omega_{P}(x)$ counting all order preserving maps $\varphi: P \rightarrow[x]$, that is,

$$
a \preceq b \quad \Longrightarrow \quad \varphi(a) \leq \varphi(b) .
$$

Here we think of $[x]=\{1,2, \ldots, x\}$ as a chain with $x$ elements, and so $\leq$ denotes the usual order in $\mathbb{R}$. The connection to chromatic polynomials is best exhibited through a variant of $\Omega_{P}(x)$, namely the number $\Omega_{P}^{\circ}(x)$ of all strictly order preserving maps $\varphi: P \rightarrow[x]$ :

$$
a \prec b \quad \Longrightarrow \quad \varphi(a)<\varphi(b) \text {. }
$$

When thinking of $P$ as an acyclic directed graph, it is a short step interpreting $\Omega_{P}^{\circ}(x)$ as a directed version of the chromatic polynomial. Along the same lines, one can write the chromatic polynomial of a given graph $G$ as

$$
\chi_{G}(x)=\sum_{\sigma \text { acyclic orientation of } G} \Omega_{\sigma}^{\circ}(x) .
$$

Stanley's two main initial results on order polynomials were

- decomposition formulas for $\Omega_{P}(x)$ and $\Omega_{P}^{\circ}(x)$ in terms of certain permutation statistics for linear extensions of $P$, from which polynomiality of $\Omega_{P}(x)$ and $\Omega_{P}^{\circ}(x)$ also follows;

- the combinatorial reciprocity theorem $(-1)^{|P|} \Omega_{P}(-x)=\Omega_{P}^{\circ}(x)$.

Date: 8 January 2019.

2000 Mathematics Subject Classification. Primary 06A07; Secondary 05A15, 05C15.

Key words and phrases. Bivariate order polynomial, bivariate chromatic polynomial, acyclic orientation, order preserving map, combinatorial reciprocity theorem. 
The latter, combined with (1), gives in turn rise to

- Stanley's reciprocity theorem for chromatic polynomials: $(-1)^{|V|} \chi_{G}(-x)$ equals the number of pairs of an $x$-coloring and a compatible acyclic orientation [7].

Our goal is to extend these three results to the bivariate setting 1 Along the way, we introduce bivariate versions of the order polynomials which, we believe, are interesting in their own right.

We will call a poset $(P, \preceq)$ bicolored if we think of $P$ as the disjoint union of $C$ and $S$, which we call the celeste and silver elements of $P$. We call $\varphi: P \rightarrow[x]$ an order preserving $(x, y)$-map if

$$
a \preceq b \Longrightarrow \varphi(a) \leq \varphi(b) \text { for all } a, b \in P \quad \text { and } \quad \varphi(c) \geq y \text { for all } c \in C .
$$

Moreover, $\varphi: P \rightarrow[x]$ is a strictly order preserving $(x, y)$-map if

$$
a \prec b \Longrightarrow \varphi(a)<\varphi(b) \text { for all } a, b \in P \quad \text { and } \quad \varphi(c)>y \text { for all } c \in C .
$$

The functions $\Omega_{P, C}(x, y)$ and $\Omega_{P, C}^{\circ}(x, y)$ count the number of order preserving $(x, y)$-maps and strictly order preserving $(x, y)$-maps, respectively. We note that these are natural extensions of the single-variable order polynomials, as $\Omega_{P}(x)=\Omega_{P, C}(x, 1)$ and $\Omega_{P}^{\circ}(x)=\Omega_{P, C}^{\circ}(x, 0)$. It is a priori not clear why $\Omega_{P, C}(x, y)$ and $\Omega_{P, C}^{\circ}(x, y)$ are polynomials in two variables, but the following simple example gives an indication.

Example 1. Consider the bicolored chain $P=\left\{a_{1}, a_{2}\right\}$ where $a_{1} \prec a_{2}$ and $a_{2}$ is celeste. We would like to find all possible strictly order preserving $(x, y)$-maps $\varphi: P \rightarrow[x]$ such that $\varphi\left(a_{2}\right)>y$. The first case

$$
1 \leq \varphi\left(a_{1}\right) \leq y<\varphi\left(a_{2}\right) \leq x
$$

gives $y$ choices for $\varphi\left(a_{1}\right)$ and $x-y$ choices for $\varphi\left(a_{2}\right)$, whereas the second case

$$
1 \leq y<\varphi\left(a_{1}\right)<\varphi\left(a_{2}\right) \leq x
$$

yields $\left(\begin{array}{c}x-y \\ 2\end{array}\right)$ choices. Therefore $\Omega_{P, C}^{\circ}(x, y)=\frac{1}{2}\left(x^{2}-x-y^{2}-y\right)$.

We now give an overview of the main results in this paper. After setting up machinery for bicolored chains in Section 2, we will prove decomposition formulas for $\Omega_{P, C}(x, y)$ and $\Omega_{P, C}^{\circ}(x, y)$ in terms of linear extensions of $P$ in Section 3 (see Theorem 7 below), as well as:

Theorem 1. The functions $\Omega_{P, C}(x, y)$ and $\Omega_{P, C}^{\circ}(x, y)$ are polynomials in $x$ and $y$. They satisfy the reciprocity theorem

$$
(-1)^{|P|} \Omega_{P, C}^{\circ}(-x,-y)=\Omega_{P, C}(x, y+1) .
$$

To state our reciprocity theorem for bivariate chromatic polynomials, which we will prove in Section 4, we recall that a flat of a graph $G$ is a graph $H$ that can be constructed from $G$ by a series of contractions. We denote by $V(H)$ the vertex set of $H$, and by $C(H)$ the set of vertices of $H$ that resulted from contractions of $G$.

Theorem 2. For any graph $G=(V, E)$,

$$
\chi_{G}(-x,-y)=\sum_{H \text { flat of } G}(-1)^{|V(H)|} m_{H}(\sigma, c),
$$

where $m_{H}(\sigma, c)$ is the number of pairs $(\sigma, c)$ consisting of an acyclic orientation $\sigma$ of $H$ and a compatible coloring $c: V(H) \rightarrow[x]$ such that $(v)>y$ if $v \in C(H)$.

Here an orientation and a coloring $c$ are compatible if $c(v) \leq c(w)$ for anyx edge oriented from $v$ to $w$.

\footnotetext{
${ }^{1}$ A reciprocity theorem for the bivariate chromatic polynomial was previously given in [2]; unfortunately, its statement and proof are wrong.
} 


\section{BICOLORED CHAINS}

Lemma 3. If $P=\left\{a_{1} \prec \cdots \prec a_{k} \prec a_{k+1} \prec \cdots \prec a_{n}\right\}$ is a bicolored chain of length $n$ where $a_{k+1}$ is the minimal celeste element, then

$$
\Omega_{P, C}^{\circ}(x, y)=\sum_{i=0}^{k}\left(\begin{array}{l}
y \\
i
\end{array}\right)\left(\begin{array}{c}
x-y \\
n-i
\end{array}\right) \quad \text { and } \quad \Omega_{P}(x, y)=\sum_{i=0}^{k}\left(\begin{array}{c}
y-2+i \\
i
\end{array}\right)\left(\begin{array}{c}
x-y+n-i \\
n-i
\end{array}\right) .
$$

Proof. We start with the count of strictly order preserving maps $\varphi: P \rightarrow[x]$ with $\varphi\left(a_{k+1}\right)>y$. This gives us a set of inequalities for $\varphi$ with $k+1$ cases: for

$$
1 \leq \varphi\left(a_{1}\right)<\cdots<\varphi\left(a_{k}\right) \leq y<\varphi\left(a_{k+1}\right)<\cdots<\varphi\left(a_{n}\right) \leq x
$$

there are $\left(\begin{array}{l}y \\ k\end{array}\right)\left(\begin{array}{l}x-y \\ n-k\end{array}\right)$ possible maps $\varphi$. The second case

$$
1 \leq \varphi\left(a_{1}\right)<\cdots<\varphi\left(a_{k-1}\right) \leq y<\varphi\left(a_{k}\right)<\cdots<\varphi\left(a_{n}\right) \leq x
$$

yields $\left(\begin{array}{c}y \\ k-1\end{array}\right)\left(\begin{array}{c}x-y \\ n-k+1\end{array}\right)$ possible maps $\varphi$. We repeat this process up to the last case

$$
1 \leq y<\varphi\left(a_{1}\right)<\cdots<\varphi\left(a_{n}\right) \leq x
$$

for which there are $\left(\begin{array}{c}y \\ 0\end{array}\right)\left(\begin{array}{c}x-y \\ n\end{array}\right)$ possible maps $\varphi$.

The count of the weakly order preserving maps $\varphi: P \rightarrow[x]$ with $\varphi\left(a_{k+1}\right) \geq y$ follows in a similar fashion.

We remark that this lemma proves polynomiality of $\Omega_{P, C}^{\circ}(x, y)$ and $\Omega_{P, C}(x, y)$, and we obtain the following precursor of Theorem 1 for chains.

Corollary 4. If $P=\left\{a_{1} \prec \cdots \prec a_{k} \prec a_{k+1} \prec \cdots \prec a_{n}\right\}$ is a bicolored chain of length $n$ where $a_{k+1}$ is the minimal celeste element, then

$$
(-1)^{n} \Omega_{P, C}^{\circ}(-x,-y)=\Omega_{P, C}(x, y+1) .
$$

Proof. By Lemma 3 and the elementary identity $(-1)^{d}\left(\begin{array}{c}-n \\ d\end{array}\right)=\left(\begin{array}{c}n+d-1 \\ d\end{array}\right)$,

$$
\begin{aligned}
\Omega_{P, C}^{\circ}(-x,-y) & =\sum_{i=0}^{k}\left(\begin{array}{c}
-y \\
i
\end{array}\right)\left(\begin{array}{c}
-x+y \\
n-i
\end{array}\right) \\
& =\sum_{i=0}^{k}(-1)^{i}\left(\begin{array}{c}
y+i-1 \\
i
\end{array}\right)(-1)^{n-i}\left(\begin{array}{c}
x-y+n-i-1 \\
n-i
\end{array}\right) \\
& =(-1)^{n} \Omega_{P, C}(x, y+1) .
\end{aligned}
$$

Next we need a refinement of the above results for certain chains that arise from a given general poset on $n$ elements. A linear extension of the bicolored poset $P$ is a chain $L$ whose underlying set (including the celeste/silver partition) equals that of $P$ such that

$$
a \preceq_{P} b \quad \Longrightarrow \quad a \preceq_{L} b .
$$

Similar to the situation in classical poset theory, linear extensions are best recorded as permutations of a fixed labeling $\omega: P \rightarrow[n]$. Namely, to a given linear extension $L=\left\{a_{1} \prec a_{2} \prec \cdots \prec a_{n}\right\}$ of $P$, we associate the word $\omega_{L}:=\omega\left(a_{1}\right) \omega\left(a_{2}\right) \cdots \omega\left(a_{n}\right)$. We collect the ascents and descents of the word $\omega_{L}$ in the sets

$$
\operatorname{Asc}\left(\omega_{L}\right):=\left\{j: \omega\left(a_{j}\right)<\omega\left(a_{j+1}\right)\right\} \quad \text { and } \quad \operatorname{Des}\left(\omega_{L}\right):=\left\{j: \omega\left(a_{j}\right)>\omega\left(a_{j+1}\right)\right\}
$$

respectively. The cardinality of these sets are denoted by $\operatorname{asc}\left(\omega_{L}\right):=\left|\operatorname{Asc}\left(\omega_{L}\right)\right|$ and $\operatorname{des}\left(\omega_{L}\right):=\left|\operatorname{Des}\left(\omega_{L}\right)\right|$, respectively.

An order preserving $(x, y)$-map $\varphi$ of $L$ is of type $\omega_{L}$ if for $i \in \operatorname{Asc}\left(\omega_{L}\right)$ we have $\varphi\left(a_{i}\right) \leq \varphi\left(a_{i+1}\right)$, and for $i \in \operatorname{Des}\left(\omega_{L}\right)$ we have $\varphi\left(a_{i}\right)<\varphi\left(a_{i+1}\right)$. Let $\Omega_{\omega_{L}}(x, y)$ denote the number of type- $\omega_{L}$ order preserving 
$(x, y)$-maps; note that this implies that $\varphi(c) \geq y$ for the minimal celeste element $c \in L$. The definition of $\Omega_{\omega_{L}}^{\circ}(x, y)$ is identical except that now $\varphi(c)>y$.

Example 2. Consider the poset $P$ and labeling $\omega$ in Figure 1 . Its three linear extensions $L_{1}, L_{2}$, and $L_{3}$ come with the associated words $\omega_{L_{1}}=12345, \omega_{L_{2}}=12435$, and $\omega_{L_{3}}=14235$, respectively.

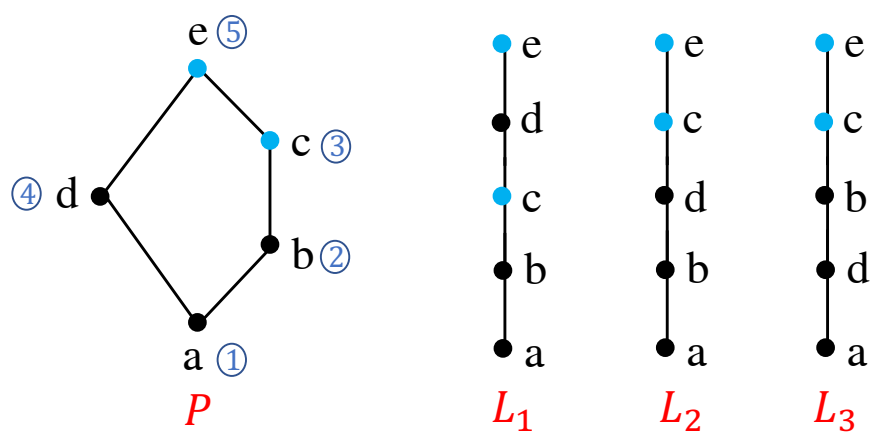

FIgURE 1. A bicolored poset $P$ with labeling $\omega$ and its linear extensions.

As an example, we will compute the number of type- $\omega_{L_{3}}$ order preserving $(x, y)$-maps $\varphi$. The word $\omega_{L_{3}}=14235$ has a descent at $i=2$. Thus, a type-14235 order preserving $(x, y)$-map satisfies the set of inequalities

$$
1 \leq \varphi(a) \leq \varphi(d)<\varphi(b) \leq \varphi(c) \leq \varphi(e) \leq x .
$$

We remove equalities by defining a bijection $\varphi \mapsto \bar{\varphi}$ via

$$
\begin{aligned}
& \bar{\varphi}(a)=\varphi(a) \\
& \bar{\varphi}(d)=\varphi(d)+\operatorname{asc}(14)=\varphi(d)+1 \\
& \bar{\varphi}(b)=\varphi(b)+\operatorname{asc}(142)=\varphi(b)+1 \\
& \bar{\varphi}(c)=\varphi(c)+\operatorname{asc}(1423)=\varphi(c)+2 \\
& \bar{\varphi}(e)=\varphi(e)+\operatorname{asc}(14235)=\varphi(e)+3 .
\end{aligned}
$$

Now (2) becomes

$$
1 \leq \bar{\varphi}(a)<\bar{\varphi}(d)<\bar{\varphi}(b)<\bar{\varphi}(c)<\bar{\varphi}(e) \leq x+3 .
$$

The additional inequality $\varphi(c)>y$ gives rise to $\bar{\varphi}(c)>y+2$. Lemma 3 now yields

$$
\Omega_{14235}^{\circ}(x, y)=\sum_{i=0}^{2}\left(\begin{array}{c}
y-2 \\
i
\end{array}\right)\left(\begin{array}{c}
x-3-y+2 \\
5-i
\end{array}\right) .
$$

This example contains essentially all ingredients needed for the proof of our next lemma.

Lemma 5. Let $P$ be a bicolored poset with a fixed labeling $\omega: P \rightarrow[n]$. Given a linear extension $L=\left\{a_{1} \prec\right.$ $\left.a_{2} \prec \cdots \prec a_{n}\right\}$ of $P$ with associated word $\omega_{L}=\omega\left(a_{1}\right) \cdots \omega\left(a_{n}\right)$, let $a_{k+1}$ be the minimal celeste element in $L$ and denote $\widetilde{\omega}_{L}=\omega\left(a_{1}\right) \cdots \omega\left(a_{k+1}\right)$. Then

$$
\Omega_{\omega_{L}}^{\circ}(x, y)=\sum_{i=0}^{k}\left(\begin{array}{c}
y-\operatorname{asc}\left(\widetilde{\omega}_{L}\right) \\
i
\end{array}\right)\left(\begin{array}{c}
x-y+\operatorname{asc}\left(\widetilde{\omega}_{L}\right)-\operatorname{asc}\left(\omega_{L}\right) \\
n-i
\end{array}\right)
$$

and

$$
\Omega_{\omega_{L}}(x, y)=\sum_{i=0}^{k}\left(\begin{array}{c}
y-\operatorname{des}\left(\widetilde{\omega}_{L}\right)-2+i \\
i
\end{array}\right)\left(\begin{array}{c}
x-y+\operatorname{des}\left(\widetilde{\omega}_{L}\right)-\operatorname{des}\left(\omega_{L}\right)+n-i \\
n-i
\end{array}\right) .
$$


Proof. We want to count the number of order preserving $(x, y)$-maps $\varphi: L \rightarrow[x]$ of type $\omega_{L}$. The map $\varphi$ satisfies the set of inequalities $\varphi\left(a_{i}\right) \leq \varphi\left(a_{i+1}\right)$ if $i$ is an ascent of $\omega_{L}$, and $\varphi\left(a_{i}\right)<\varphi\left(a_{i+1}\right)$ if $i$ is a descent of $\omega_{L}$. We eliminate weak inequalities by creating a bijection $\varphi \rightarrow \bar{\varphi}$ defined by $\bar{\varphi}\left(a_{1}\right)=\varphi\left(a_{1}\right)$ and

$$
\bar{\varphi}\left(a_{i}\right)=\varphi\left(a_{i}\right)+\operatorname{asc}\left(\omega\left(a_{1}\right) \cdots \omega\left(a_{i}\right)\right) \quad \text { for } 2 \leq i \leq n .
$$

If $i \in \operatorname{Des}\left(\omega_{L}\right)$, then $\operatorname{asc}\left(\omega\left(a_{1}\right) \cdots \omega\left(a_{i+1}\right)\right)-\operatorname{asc}\left(\omega\left(a_{1}\right) \cdots \omega\left(a_{i}\right)\right)=0$, and so

$$
\varphi\left(a_{i}\right)<\varphi\left(a_{i+1}\right) \quad \Longrightarrow \quad \bar{\varphi}\left(a_{i}\right)<\bar{\varphi}\left(a_{i+1}\right) .
$$

If $i \in \operatorname{Asc}\left(\omega_{L}\right)$, then $\operatorname{asc}\left(\omega\left(a_{1}\right) \cdots \omega\left(a_{i+1}\right)\right)-\operatorname{asc}\left(\omega\left(a_{1}\right) \cdots \omega\left(a_{i}\right)\right)=1$, whence

$$
\varphi\left(a_{i}\right) \leq \varphi\left(a_{i+1}\right) \quad \Longrightarrow \quad \bar{\varphi}\left(a_{i}\right)<\bar{\varphi}\left(a_{i+1}\right) .
$$

In addition, $\varphi\left(a_{k+1}\right)>y$ implies $\bar{\varphi}\left(a_{k+1}\right)>y+\operatorname{asc}\left(\widetilde{\omega}_{L}\right)$, and with

$$
1 \leq \bar{\varphi}\left(a_{1}\right)<\cdots<\bar{\varphi}\left(a_{n}\right) \leq x+\operatorname{asc}\left(\omega_{L}\right),
$$

the formula for $\Omega_{\omega_{L}}^{\circ}(x, y)$ now follows from Lemma 3 .

The formula for $\Omega_{\omega_{L}}(x, y)$ follows in an analogous fashion, replacing all ascents in our argumentation by descents: now our bijection is defined via $\bar{\varphi}\left(a_{1}\right)=\varphi\left(a_{1}\right)$ and

$$
\bar{\varphi}\left(a_{i}\right)=\varphi\left(a_{i}\right)-\operatorname{des}\left(\omega\left(a_{1}\right) \cdots \omega\left(a_{i}\right)\right) \quad \text { for } 2 \leq i \leq n,
$$

giving rise to $\bar{\varphi}\left(a_{k+1}\right) \geq y+\operatorname{des}\left(\widetilde{\omega}_{L}\right)$ and

$$
1 \leq \bar{\varphi}\left(a_{1}\right) \leq \cdots \leq \bar{\varphi}\left(a_{n}\right) \leq x+\operatorname{des}\left(\omega_{L}\right) .
$$

The inverse of $\omega=\omega_{1} \cdots \omega_{n}$ is the word $\bar{\omega}$ defined by $\bar{\omega}_{j}:=\omega_{n+1-j}$. This switches all ascents with descents; in particular, $\operatorname{asc}\left(\omega_{L}\right)=\operatorname{des}\left(\bar{\omega}_{L}\right)$ and $\operatorname{des}\left(\omega_{L}\right)=\operatorname{asc}\left(\bar{\omega}_{L}\right)$.

Corollary 6. Let $P$ be a bicolored poset with a fixed labeling $\omega: P \rightarrow[n]$, and let $L=\left\{a_{1} \prec a_{2} \prec \cdots \prec a_{n}\right\}$ be a linear extension of $P$, with associated word $\omega_{L}=\omega\left(a_{1}\right) \cdots \omega\left(a_{n}\right)$. Then

$$
(-1)^{n} \Omega_{\omega_{L}}^{\circ}(-x,-y)=\Omega_{\bar{\omega}_{L}}(x, y+1) .
$$

Proof. Let $a_{k+1}$ be the minimal celeste element in $L$ and let $\widetilde{\omega}_{L}=\omega\left(a_{1}\right) \cdots \omega\left(a_{k+1}\right)$. By Lemma 5 and the fact that $\operatorname{asc}\left(\widetilde{\omega}_{L}\right)=\operatorname{des}\left(\widetilde{\widetilde{\omega}}_{L}\right)$,

$$
\begin{aligned}
\Omega_{\omega_{L}}^{\circ}(-x,-y) & =\sum_{i=0}^{k}\left(\begin{array}{c}
-y+\operatorname{asc}\left(\widetilde{\omega}_{L}\right) \\
i
\end{array}\right)\left(\begin{array}{c}
-x+y+\operatorname{asc}\left(\widetilde{\omega}_{L}\right)-\operatorname{asc}\left(\omega_{L}\right) \\
n-i
\end{array}\right) \\
& =\sum_{i=0}^{k}(-1)^{i}\left(\begin{array}{c}
y-\operatorname{asc}\left(\widetilde{\omega}_{L}\right)+i-1 \\
i
\end{array}\right)(-1)^{n-i}\left(\begin{array}{c}
x-y-\operatorname{asc}\left(\widetilde{\omega}_{L}\right)+\operatorname{asc}\left(\omega_{L}\right)+n-i-1 \\
n-i
\end{array}\right) \\
& =(-1)^{n} \sum_{i=0}^{k}\left(\begin{array}{c}
y-\operatorname{des}\left(\widetilde{\widetilde{\omega}}_{L}\right)+i-1 \\
i
\end{array}\right)\left(\begin{array}{c}
x-y-1-\operatorname{des}\left(\widetilde{\widetilde{\omega}}_{L}\right)+\operatorname{des}\left(\bar{\omega}_{L}\right)+n-i \\
n-i
\end{array}\right) \\
& =(-1)^{n} \Omega_{\bar{\omega}_{L}}(x, y+1) .
\end{aligned}
$$

\section{Bicolored Posets}

To apply Lemma 5 to general posets, we recall the notions of a natural labeling $\omega: P \rightarrow[n]$ of a poset $P$ with $n$ elements, that is,

and a reverse natural labeling:

$$
a \prec b \quad \Longrightarrow \quad \omega(a)<\omega(b),
$$

$$
a \prec b \quad \Longrightarrow \quad \omega(a)>\omega(b) .
$$


Theorem 7. Let $P$ be a bicolored poset with a reverse natural labeling $\omega$ and a natural labeling $\tau$. Then

$$
\Omega_{P, C}^{\circ}(x, y)=\sum_{L} \Omega_{\omega_{L}}^{\circ}(x, y) \quad \text { and } \quad \Omega_{P, C}(x, y)=\sum_{L} \Omega_{\tau_{L}}(x, y)
$$

where both sums are over all possible linear extensions $L$ of $P$.

Proof. We will show that there is a bijection between the set of strictly order preserving $(x, y)$-maps $\varphi$ and the set of all pairs $(L, \hat{\varphi})$ where $L$ is a linear extension and $\hat{\varphi}$ is a type- $\omega_{L}$ map on the bicolored poset $P$.

Suppose $\varphi$ is a strictly order preserving $(x, y)$-map. We will build a linear extension $L$ for which $\varphi$ is of type $\omega_{L}$.

- If $\varphi\left(a_{i}\right)<\varphi\left(a_{j}\right)$, then let $a_{i} \prec a_{j}$ in $L$. Note that $\omega\left(a_{i}\right)>\omega\left(a_{j}\right)$ because $\omega$ is a natural reverse labeling.

- If $\varphi\left(a_{i}\right)=\varphi\left(a_{j}\right)$ with $\omega\left(a_{i}\right)<\omega\left(a_{j}\right)$, then let $a_{i} \prec a_{j}$ in $L$.

- If $\varphi\left(a_{i}\right)=\varphi\left(a_{j}\right)$ with $\omega\left(a_{i}\right)>\omega\left(a_{j}\right)$, then let $a_{i} \succ a_{j}$ in $L$.

This gives us the linear extension $L$ with associated word $\omega_{L}$, and by construction, $\varphi$ is of type $\omega_{L}$.

Conversely, let $L$ be a linear extension of $P$ and let $\hat{\varphi}$ be a type- $\omega_{L}$ map. Type- $\omega_{L}$ maps agree with strictly order preserving maps between comparable elements in a poset by definition. The map $\hat{\varphi}$ may give us weak or strict inequalities between incomparable elements in $P$. Strictly order preserving maps place no restrictions on what happens between incomparable elements in the poset. Thus, $\hat{\varphi}$ is also a strictly order preserving map on $P$.

The proof of the second part is similar to that of the first part with one major difference: now we have $\tau\left(a_{i}\right)<\tau\left(a_{j}\right)$ when $a_{i} \prec a_{j}$ in the linear extension $L$ because $\tau$ is a natural labeling.

Proof of Theorem 1 Polynomiality follows from Lemma 5 and Theorem 7 .

To prove the reciprocity statement, fix a natural reverse labeling $\omega$ of $P$; note that $\bar{\omega}$ is a natural labeling. By Corollary 6 and Theorem 7 ,

$$
\Omega_{P}^{\circ}(-x,-y)=\sum_{L} \Omega_{\omega_{L}}^{\circ}(-x,-y)=(-1)^{n} \sum_{L} \Omega_{\bar{\omega}_{L}}(x, y+1)=(-1)^{n} \Omega_{P}(x, y+1) .
$$

\section{Bivariate Chromatic Polynomials}

In preparation for our proof of Theorem 2, we show the following decomposition result.

\section{Lemma 8.}

$$
\chi_{G}(x, y)=\sum_{H \text { flat of } G} \sum_{\begin{array}{c}
\sigma \text { acyclic } \\
\text { orientation of } H
\end{array}} \Omega_{\sigma, C(H)}^{\circ}(x, y) .
$$

Proof. Let $c: V \rightarrow[x]:=\{1,2, \ldots, x\}$ be a coloring of $G$ that satisfies

$$
c(v) \neq c(w) \quad \text { or } \quad c(v)=c(w)>y .
$$

Let $H$ be the flat of $G$ obtained by contracting all edges whose endpoints have the same color; note that the colors of $C(H)$ will be $>y$. We orient the edges of $H$ by their color gradient: $v \rightarrow u$ if $c(v)<$ $c(u)$. The resulting orientation will be acyclic, and the resulting colorings of $H$ will precisely be counted by $\Omega_{\sigma, C(H)}^{\circ}(x, y)$.

This argumentation can be reversed: given a flat $H$ of $G$ and an acyclic orientation $\sigma$ of $H$, a strictly order preserving $(x, y)$-map counted by $\Omega_{\sigma, C(H)}^{\circ}(x, y)$ can be extended to a coloring of $G$ where vertices that result in contractions for $H$ get colors $>y$. 

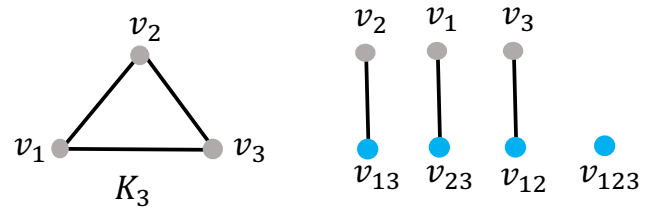

FIGURE 2. Contractions of $K_{3}$.

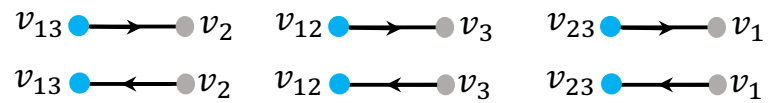

FIGURE 3. Acyclic orientations of contractions of $K_{3}$.

Example 3. Our proof of Lemma 8 is illustrated for the case $G=K_{3}$ in Figures 2 and 3 .

For $H=G$ we obtain $\Omega_{\sigma, \varnothing}^{\circ}(x, y)=\left(\begin{array}{l}x \\ 3\end{array}\right)$ for any acyclic orientation $\sigma$, and summing over all six such orientations gives the univariate chromatic polynomial of $K_{3}$.

If $H$ is the result of one contraction, we need to consider two orientations $\sigma_{1}$ and $\sigma_{2}$ with order polynomials

$$
\Omega_{\sigma_{1}, v_{12}}^{\circ}(x, y)=\left(\begin{array}{c}
x-y \\
2
\end{array}\right)+(x-y) y \quad \text { and } \quad \Omega_{\sigma_{2}, v_{12}}^{\circ}(x, y)=\left(\begin{array}{c}
x-y \\
2
\end{array}\right) .
$$

Finally, if $H$ results from contracting two edges, $\Omega_{\sigma, v_{123}}^{\circ}(x, y)=x-y$. Thus

$$
\chi_{K_{3}}(x, y)=6\left(\begin{array}{l}
x \\
3
\end{array}\right)+3\left(\left(\begin{array}{c}
x-y \\
2
\end{array}\right)+(x-y) y+\left(\begin{array}{c}
x-y \\
2
\end{array}\right)\right)+x-y=x^{3}-3 x y+y .
$$

Proof of Theorem 2 By Theorem 1 and Lemma 8 ,

$$
\chi_{G}(-x,-y)=\sum_{H \text { flat of } G} \sum_{\begin{array}{c}
\sigma \text { acyclic } \\
\text { orientation of } H
\end{array}}(-1)^{|V(H)|} \Omega_{\sigma, C(H)}(x, y+1) .
$$

Here $\Omega_{\sigma, C(H)}(x, y+1)$ counts the number of order preserving maps $\varphi: \sigma \rightarrow[x]$ subject to two conditions: First, if $c \in C(H)$ then $\varphi(c) \geq y+1$; and second, $\varphi$ is compatible with $\sigma$.

\section{REFERENCES}

1. Ilia Averbouch, Benny Godlin, and Johann A. Makowsky, An extension of the bivariate chromatic polynomial, European J. Combin. 31 (2010), no. 1, 1-17.

2. Matthias Beck and Mela Hardin, A bivariate chromatic polynomial for signed graphs, Graphs Combin. 31 (2015), no. 5, 1211-1221, arXiv: 1204.2568.

3. Klaus Dohmen, Closed-form expansions for the universal edge elimination polynomial, Australas. J. Combin. 63 (2015), $196-201$.

4. Klaus Dohmen, André Pönitz, and Peter Tittmann, A new two-variable generalization of the chromatic polynomial, Discrete Math. Theor. Comput. Sci. 6 (2003), no. 1, 69-89.

5. Christopher J. Hillar and Troels Windfeldt, Fibonacci identities and graph colorings, Fibonacci Quart. 46/47 (2008/09), no. 3, 220-224, arXiv:0805.0992.

6. Richard P. Stanley, A chromatic-like polynomial for ordered sets, Proc. Second Chapel Hill Conf. on Combinatorial Mathematics and its Applications (Univ. North Carolina, Chapel Hill, N.C., 1970), Univ. North Carolina, Chapel Hill, N.C., 1970, pp. $421-427$.

7. __ Acyclic orientations of graphs, Discrete Math. 5 (1973), 171-178.

8. __ Enumerative Combinatorics. Volume 1, second ed., Cambridge Studies in Advanced Mathematics, vol. 49, Cambridge University Press, Cambridge, 2012. 
Department of Mathematics, San Francisco State University, San Francisco, CA 94132, U.S.A.

E-mail address: mattbeck@sf su.edu

Department of Mathematics, Mills College, Oakland, CA 94613, U.S.A.

E-mail address: mf arahmand@mills.edu

College of Alameda, 555 Ralph Appezzato Memorial Parkway, Alameda, CA 94501, U.S.A.

E-mail address: gkarunaratne@peralta.edu

Graduate School of Education, University of CAlifornia-Berkeley, Berkeley, CA 94720, U.S.A.

E-mail address: zuni5344@berkeley.edu 